\title{
L'appartenance religieuse dans la jurisprudence de la Cour de Strasbourg
}

Daniele Ferrari

\section{OpenEdition}

12 Journals

Édition électronique

URL : http://journals.openedition.org/rdr/1136

DOI : $10.4000 /$ rdr.1136

ISSN : 2534-7462

Éditeur

Presses universitaires de Strasbourg

\section{Édition imprimée}

Date de publication : 9 juillet 2020

Pagination : 125-141

ISBN : 979-10-344-0065-2

ISSN : 2493-8637

Référence électronique

Daniele Ferrari, «L'appartenance religieuse dans la jurisprudence de la Cour de Strasbourg », Revue du droit des religions [En ligne], 9 | 2020, mis en ligne le 18 mai 2020, consulté le 19 novembre 2020. URL: http://journals.openedition.org/rdr/1136; DOI : https://doi.org/10.4000/rdr.1136

\section{(c) $(1)(9$}

La revue du droit des religions est mise à disposition selon les termes de la Creative Commons Attribution - Pas d'Utilisation Commerciale 4.0 International - CC BY-NC 4.0. 


\section{L'APPARTENANCERELIGIEUSE DANSLA JURISPRUDENCE DELACOURDESTRASBOURG}

\section{Daniele FERRARI}

Postdoctorant, Università degli Studi di Siena

\section{RÉSUMÉ}

Cette étude est une réflexion sur la relation entre appartenance et protection de la liberté de conscience et de religion à la lumière de l'interprétation de l'article 9 de la Convention européenne des droits de l'homme par la Cour de Strasbourg. La question s'articule autour de l'individualisme moderne en matière religieuse et de la position des autorités (administratives ou judiciaires) face à une demande de liberté religieuse non régulée par les communautés religieuses.

\section{Abstract}

This paper proposes a reflection on the relationship between belonging and protection of freedom of conscience and religion in the light of the interpretation of Article 9 of the European Convention on Human Rights by the Strasbourg Court. The issue revolves around modern individualism in religious matters and the position of the authorities (administrative or judicial) when faced with a demand for religious freedom that is not regulated by religious communities. 
$\mathrm{C}$ ette étude propose une réflexion sur le rapport entre appartenance religieuse et protection de la liberté de conscience et de religion au vu de l'interprétation de l'article 9 de la Convention européenne des droits de l'homme (CEDH) adoptée par la Cour de Strasbourg. La problématique tourne autour de l'individualisme moderne en matière religieuse et de la position des autorités (administratives ou judiciaires) face à une revendication de liberté religieuse non régulée par les communautés religieuses.

Cette réflexion présente un intérêt tant au niveau sociologique qu'au niveau de la doctrine juridique. En particulier, l'intérêt social découle de la circonstance que la manière de vivre la foi ou une conviction semble aujourd'hui se traduire, de plus en plus, par une personnalisation de la morale humaine (une sorte de "religion à la carte ${ }^{1} »$ ), à laquelle correspond un recul de la dimension collective et congrégationaliste. Ainsi, les différentes identités liées au credo ne coïncident pas nécessairement avec l'appartenance à un groupe, formalisée par exemple par des actes spécifiques d'enregistrement confessionnel (que l'on pense aux registres de baptême), des règles ou des pratiques codifiées et reconnues ou reconnaissables au sein des différentes traditions identifiables dans les États européens ${ }^{2}$.

D'un point de vue juridique, la doctrine française a récemment étudié le rapport entre une personne et une communauté confessionnelle, opposant le concept d'affiliation à celui d'appartenance religieuse ${ }^{3}$ : le premier est considéré comme un vestige du passé, correspondant à une conception de la religion comme imposition, tandis que le second au sens moderne est qualifié de manifestation du libre choix de la personne, qui décide de manière indépendante si et à quelle confession religieuse adhérer.

Partant de ces prémisses, cet article propose une reconstruction du concept juridique d'appartenance religieuse dans la jurisprudence de la Cour de Strasbourg à travers: une introduction à la genèse historique de cette notion en droit international; le développement de ce concept par la Cour européenne des droits de l'homme.

En droit international, le rapport entre état civil, appartenance nationale et appartenance religieuse peut être configuré suivant trois modèles principaux

1. V. J.-L. Schlegel, Religions à la carte, Paris, Fayard, 2012.

2. V. C. Mirabelli, L'appartenenza confessionale, Padova, CEDAM, 1975, p. 250 et s.; Y. LAMBERT, "Des changements dans l'évolution religieuse de l'Europe et de la Russie», Rev. fr. sociologie, $\mathrm{n}^{\circ}$ 2004/2, p. 307-338.

3. V. F. MESSNER, «Introduction. L'affiliation religieuse en Europe», in F. MESSNER (dir.), L'affiliation religieuse en Europe, Strasbourg, Presses universitaires de Strasbourg, 2017, p. 5 et s. 
d'appréhension de la conscience, qui se sont succédé dans le temps: a) la conscience comme manifestation du devoir du sujet de croire en la religion du souverain selon le modèle cujus regio ejus religio; b) la conscience comme expression du lien entre statut civil et statut religieux à l'étranger ou dans un territoire défini (protection de la liberté religieuse des étrangers ou des groupes minoritaires) ${ }^{4}$; c) la conscience comme appartenance de l'individu à l'humanité (le statut de l'homme de la Déclaration universelle de 1948).

Dans l'évolution historique des systèmes politiques, le chevauchement entre le statut civil et le statut religieux a qualifié la conscience non pas comme une liberté de l'individu, mais comme l'acceptation d'une vérité supérieure qui a coïncidé avec la religion qui, dans l'histoire de l'Europe, a été imposée par les princes et empereurs aux personnes affectées à un territoire donné ${ }^{5}$. De l'édit de Thessalonique, délivré par l'empereur Théodose en 380 après J.-C. ${ }^{6}$, jusqu'à la Réforme protestante ${ }^{7}$, le principe d'ordre sur lequel se construisent les règles de droit est figuré par le christianisme ${ }^{8}$.

Dans un premier temps, le détachement entre status religionis et status civilis se définit à partir de la reconnaissance internationale de la liberté de religion ${ }^{9}$. En particulier, à partir du XVII ${ }^{\mathrm{e}}$ siècle ${ }^{10}$, la liberté religieuse est bien établie en droit international, dans le but de protéger des groupes religieux spécifiques ${ }^{11}$.

4. V. A. Pizzorusso, Le minoranze nel diritto pubblico interno, Milano, Giuffrè, 1967, p. 21 et s.; B. NAsCimbene, Il trattamento dello straniero nel diritto internazionale ed europeo, Milano, Giuffrè, 1984, p. 25 et s.

5. V. F. Ruffini, Relazioni tra Stato e Chiesa, Bologna, Il Mulino, 1974, p. 67.

6. Avec Théodose se définit un «mariage définitif de l'Empire romain et de l'Église chrétienne»: G. Lagarde, La naissance de l'esprit laïque au déclin du Moyen Âge, t. 1, Paris, Armand Colin, 13éd. 1973, p. 14.

7. V. A. Pizzorusso, op.cit., p. 15. V. sur la Réforme protestante, M. Biagioni, L. Felici, La Riforma radicale nell'Europa del Cinquecento, Roma-Bari, Laterza, 2012.

8. V. E. W. BöCKENFÖRDE, Diritto e secolarizzazione. Dallo Stato moderno all'Europa unita, G. Preterossi (trad.), Roma, Laterza, 2007, p. 36-37.

9. L. BRESSAN, Libertà religiosa nel diritto internazionale. Dichiarazioni e norme internazionali, Padova, CEDAM, 1989, p. 19 et s.

10. Ce n'est pas un hasard si la Paix de Westphalie de 1648, qui met fin à la guerre de Trente Ans, oblige les souverains à respecter des groupes religieux spécifiques.

11. Sur ce point, la doctrine a parlé de «règles internationales protégeant la liberté de religion des sujets contenus dans les traités stipulés à l'occasion des transferts territoriaux» : G. Balladore Pallieri, Il diritto internazionale ecclesiastico, Padova, CEDAM, 1940, p. 143. Par exemple, des dispositions relatives à la protection de la liberté religieuse des sujets de confessions différentes de celle du nouveau souverain figurent dans le Traité de paix d'Oliva de 1660 concernant les territoires cédés par la Prusse (art. 2 \& 3) et par la Pologne (art. 4 § 2) à la Suède. 
Avec la dernière phase, qui coïncide avec la proclamation de la Déclaration universelle des droits de l'homme de 1948, l'individu n'est plus un moyen, même au sens moral, du pouvoir souverain dont il est sujet et protégé seulement s'il est étranger ou s'il fait partie d'un groupe minoritaire, mais un sujet autonome doté d'une conscience, dont le statut universel est défini par la protection de la liberté de conscience et de religion ${ }^{12}$ comme conséquence de l'appartenance au genre humain. De ce point de vue, les libertés de conscience et de religion doivent être protégées, quelle que soit leur correspondance avec des doctrines ou comportements codifiés par un groupe ou avec une expression des traditions nationales d'un État. La liberté morale, en effet, devrait toujours se justifier en un status libertatis et le critère d'appartenance être pertinent uniquement lorsque c'est l'individu qui le qualifie comme élément de sa propre identité spirituelle ou philosophique. Compte tenu de la genèse et du développement de la liberté et de la conscience religieuse, la jurisprudence de la Cour européenne des droits de l'homme offre un exemple intéressant de la manière dont la protection de ces libertés s'exprime, au prisme de l'application de l'article 9 de la CEDH, par une dynamique complexe de concurrence entre le modèle historique d'appartenance religieuse et la protection de l'autonomie de la personne. Cette dialectique sera construite à travers les concepts de croyance ou conviction, d'appartenance religieuse et du contexte national dans le cadre de la jurisprudence de la Cour européenne des droits de l'homme. Ces trois concepts sont pertinents en ce qu'ils expriment deux significations différentes de la relation entre la liberté de conscience et de religion et l'appartenance.

Premièrement, les notions de conviction et d'appartenance religieuse concernent la dimension individuelle et collective de l'appartenance. Dans le contexte des croyances ou conviction, en effet, le sens individuel d'appartenance se dégage des critères que la Cour de Strasbourg a développés pour qualifier la relation entre l'individu et sa propre conscience. L'appartenance religieuse, quant à elle, concerne les profils collectifs des croyances personnelles, c'est-à-dire le rôle que le lien entre l'individu et le groupe a dans les modèles de garantie de la liberté de conscience et de religion développés par la Cour européenne des droits de l'homme.

Deuxièmement, le contexte national à l'origine du recours peut, comme cela sera précisé en deuxième partie, refléter les décisions de la Cour de

12. V. A. CASSESE, I diritti umani nel mondo contemporaneo, Roma-Bari, La Terza, 2002, p. 41 et s.; P. Costa, «I diritti oltre lo Stato. La Dichiarazione del 1948 e la sua retorica "universalistica" », in M. Salvati (dir.), Dichiarazione universale dei diritti dell'uomo 10 dicembre 1948. Nascita, declino e sviluppi, Roma, Ediesse, $2^{\text {a }}$ ed. 2008, p. 39 et s. 
Strasbourg sur la liberté de religion. En ces termes, bien que l'article 1 de la CEDH dispose que: «Les Hautes Parties contractantes reconnaissent à toute personne relevant de leur juridiction les droits et libertés définis au titre I de la présente Convention», sous certaines conditions, le contexte national à l'origine du recours peut affecter l'application de la Convention (et des protocoles additionnels) par la Cour.

\section{APPARTENANCE ET LIBERTÉ : LA DÉFINITION DE CONVICTION ET LE RÔLE DE L'APPARTENANCE RELIGIEUSE}

La Convention européenne des droits de l'homme de 1950 a adopté le modèle de protection de la liberté de religion et de conscience de la Déclaration universelle des droits de l'homme, développé à l'échelle mondiale. L'article 9, en particulier, garantit la liberté de l'esprit autant comme protection de l'autonomie interne de la personne (forum internum) que comme protection des comportements motivés par la conscience personnelle (forum externum) qui ne peuvent être limités qu'en présence de raisons d'ordre public, de sécurité, de santé et de moralité publiques jugées nécessaires par les autorités publiques et judiciaires dans une société démocratique. Ainsi, la Convention ne fait pas référence à un modèle de conviction en particulier, et donc d'appartenance à une Église ou à une identité nationale, protégeant toutes les orientations morales ou philosophiques personnelles, qui, à moins d'être antidémocratiques, doivent être libres de s'exprimer au sein des différentes communautés de l'État. Le besoin de protéger toutes les options morales - y compris celles professées par des membres de mouvements «sectaires» ou athées ${ }^{13}$ - apparaît dans la jurisprudence, d'abord de la Commission et ensuite de la Cour qui ont refusé, par exemple, de faire la distinction entre confessions religieuses et sectes ${ }^{14}$.

13. La Cour EDH, dans l'arrêt du 25 mai 1993 Kokkinakis c. Grèce, § 31, a pu déterminer que la zone de protection tracée par l'article 9 de la Convention représente une ligne directrice fondamentale d'une société démocratique, en vertu de la même Convention. Celle-ci relève autant du niveau de la religion, en accordant une protection spécifique à l'identité des croyants et à leur conception de la vie, que du niveau du respect des athées, des agnostiques, des sceptiques ou des indifférents.

14. Ce point de vue de la Cour apparaît une première fois dans Comm. EDH, 4 oct. 1977 , $X$ c. Royaume-Uni, où elle précise que la protection des croyances religieuses n'est pas limitée aux grandes religions, mais également aux convictions religieuses qui sont identifiables. Par conséquent, la Commission n'a jamais utilisé les groupes religieux traditionnels des différents États comme modèles pour définir le concept de confession religieuse; dans le cas de la scientologie: Comm. EDH, 5 mai 1979, X et Église scientologique c. Suède; druidisme: Comm. EDH, 14 juill. 1987, Chappell c. Royaume-Uni ; «Divine Light Zentrum»: 
En outre, si l'article 9 prévoit, d'une manière générale, le droit à la liberté de conscience, quel que soit le contenu de la morale subjective, l'égalité de toutes les convictions humaines est protégée par le principe de non-discrimination (art. 14 CEDH) et cela indépendamment des modèles nationaux de relations État-religions, qui, en vertu de la Convention, ne devraient justifier aucune dérogation à la garantie des droits consacrés à l'article $9^{15}$.

Dans ce contexte, la Cour européenne des droits de l'homme, au-delà des comportements religieux traditionnels encodés dans une relation d'appartenance de l'individu à une confession religieuse, a créé une véritable définition des convictions humaines en relation avec l'article 9.

Un premier critère est énoncé dans un arrêt où le pouvoir judiciaire européen rappelle que «tous les avis ou convictions n'entrent pas dans le champ d'application de l'article $9 \S 1$ de la Convention ${ }^{16}$ », parce que «Comme l'a dit la Commission ${ }^{17}$, le terme "pratiques" employé à l'article 9 \& 1 ne recouvre pas tout acte motivé ou influencé par une religion ou une conviction». La Cour de Strasbourg en 2019, dans le Guide sur l'article 9 de la Convention européenne des droits de l'homme a réaffirmé ces arguments, en soulignant comment dans sa propre jurisprudence a été consolidé le principe selon lequel: «Pour qu'une conviction personnelle ou collective puisse relever du droit à la "liberté de pensée, de conscience et de religion", il faut qu'elle atteigne un degré suffisant de force, de sérieux, de cohérence et d'importance ${ }^{18} »$. Rebus sic stantibus, quelles pratiques pourront être garanties sur le fondement de l'article 9 de la Convention, comme manifestation d'une conviction?

Comm. EDH, 19 mars 1981, Omkarananda et le Divine Light Zentrum c. Suisse. À ces groupes minoritaires s'ajoute le mouvement raélien: CEDH, 3 nov. 2005, F. L. c. France et le mouvement fondé par Osho: CEDH, 6 nov. 2008, Leela Förderkreis E. V. c. Allemagne. V. G. GonZAlez, «Haro sur les "sectes" mais... pas trop!», RTDH 2009, p. 553-568; F. G. Jacobs, C. Ovey, R. White, The European Convention on Human Rights, Oxford, Oxford University Press, $4^{\text {th }}$ ed. 2006, p. 403 et s. et S. FERRARI, «Le droit européen en matière religieuse et ses conséquences pour les sectes», in F. Champion, M. Cohen (dir.), Sectes et démocratie, Paris, Seuil, 1999, p. 359 et s.

15. De plus, le fait que les États avec un système d'Église nationale ou privilégiée n'aient opposé, dans le cadre de la ratification, aucune réserve à l'article 9 (la seule réserve a été faite par la Norvège, qui l'a ensuite annulée en 1956) démontrait un certain consensus sur la compatibilité entre ces dispositions et la garantie de la liberté de religion et de conscience.

16. CEDH, 29 avr. 2002, Pretty c. Royaume-Uni.

17. V., entre autres, le rapport de la Commission du 12 octobre 1978, dans le cas Arrowsmith c. Royaume-Uni: D.R. 19, p. 15 et s.; CEDH, $1^{\text {er }}$ juill. 1997, Kalaç c. Turquie, § 27 ; Comm. EDH, 15 déc. 1983, C. c. Royaume-Uni: D.R. 37, p. 142 et s.

18. V. CEDH, Guide sur l'article 9 de la Convention européenne des droits de l'homme. Liberté de pensée, de conscience et de religion, 2019, p. 9 et s. : https://www.echr.coe.int/Documents/ Guide_Art_9_FRA.pdf [consulté le 20 janv. 2020]. 
Pour répondre à cette question, il faut d'abord évoquer la définition de conviction élaborée par la Cour puis reconstruire ses applications.

La définition de conviction a été initialement remise en question sans rapport avec l'interprétation de l'article 9 de la CEDH, mais avec l'article $2 \S 2$ $\mathrm{du}$ protocole $\mathrm{n}^{0} 1$ à la Convention. Dans l'affaire Campbell et Cosans c. Royaume-Uni, la Cour donne une définition de la conviction humaine. En particulier, les convictions sont des «vues atteignant un certain degré de force, de sérieux, de cohérence et d'importance ${ }^{19}$ ", se différenciant ainsi dans la protection accordée à leur manifestation en public ou en privé, de simples opinions ou idées, protégées par l'article $10 \mathrm{CEDH}$ comme formes d'expression. Les convictions sont, comme la Commission a eu l'occasion de le rappeler dans une décision antérieure ${ }^{20}$ (mais pas à travers une définition explicite comme dans l'affaire Campbell et Cosans) l'« expression d'une vision cohérente sur un problème fondamental».

$\mathrm{Au}$ niveau de l'application de la définition, le pacifisme a été considéré comme une conviction, l'expression d'une philosophie de la vie, protégée conformément à l'article 9 , bien que le terme pratique, au sens de l'article $9 \S 1$, poursuit la Cour dans cette décision, «ne désigne n'importe quel acte motivé ou inspiré par une religion ou une conviction ${ }^{21} »$. De même, le refus pour des raisons éthiques, opposé par de petits propriétaires, de transférer les droits publics sur leurs terres pour la chasse, manifeste des convictions «expressives d'une certaine force, cohérence et importance, pour les rendre dignes de respect dans une société démocratique ${ }^{22} »$. D’autres idées ont été intégrées par la jurisprudence dans le cadre des convictions de conscience, protégées par l'article $9 \mathrm{CEDH}$, comme la lutte contre l'avortement ${ }^{23}$, le véganisme ${ }^{24}$, voire dans un cas l'appartenance politique ${ }^{25}$, l'objection de conscience ${ }^{26}$.

19. CEDH, 25 févr. 1982, Campbell et Cosans c. Royaume-Uni, \& 36.

20. Comm. EDH, déc. 10 mars 1981, X c. RFA: D.R. 24, p. 141.

21. Rapport Comm. EDH, 12 oct. 1978, Arrowsmith c. Royaume-Uni, § 71. Pour d'autres exemples sur le concept de conviction selon la Convention européenne des droits de l'homme: G. Dole, La liberté d'opinion et de conscience en droit communautaire, Paris, LGDJ, 1997, p. 30 et s.; J.-P. Schouppe, La dimension institutionnelle de la liberté de religion dans la jurisprudence de la Cour européenne des droits de l'homme, Paris, Pedone, 2015.

22. CEDH, Gde ch., 29 avr. 1999, Chassagnou et autres c. France, §§ 114-123.

23. Comm. EDH., 8 mars 1985, Knudsen c. Norvège.

24. Comm. EDH, 10 févr. 1993, W. c. Royaume-Uni.

25. Même si normalement l'appartenance politique est rattachée aux articles 10 et 11 , dans le cas étudié, elle a été examinée sous l'angle de l'article 9: Comm. EDH, déc. 11 oct. 1991, Hazar et autres c. Turquie: D.R. 72, p. 200.

26. CEDH, Gde ch., 6 avr. 2000, Thlimmenos c. Grèce; CEDH, 24 janv. 2006, Ülke c. Turchia ; CEDH, Gde ch., 7 juill. 2011, Bayatyan c. Armenia; CEDH, 17 oct. 2019, Mushfig 
Ainsi, si la Cour reconnaît que la protection de la liberté de conscience, en veillant à l'objet de la motivation (vision cohérente sur un problème fondamental), se fonde sur une conduite, ainsi que sur la force de la conviction, sa fiabilité, sa cohérence et sa pertinence, il reste à examiner les outils utilisés pour son appréciation.

Venant au concept d'appartenance religieuse, le jugement sur les convictions personnelles peut s'appuyer sur un critère d'autoqualification qui, tout en protégeant l'autonomie de la conscience, est fondé sur l'évaluation que le sujet donne aux actions en les classant comme expression de ses convictions ou sur un critère d'hétéronomie ${ }^{27}$.

En suivant le premier critère, le juge peut élargir l'effet juridique de protection d'un comportement, conformément à l'article 9, assurant la liaison entre la conduite et la conviction, sur la base de l'évaluation donnée par l'individu. Cette orientation peut déjà se retrouver dans certaines opinions dissidentes, dans lesquelles on soutenait, conformément au principe d'autoqualification, que la manière dont les individus perçoivent leurs options de conscience «doit être acceptée par la Cour, sauf si elle est manifestement infondée ou irrationnelle ${ }^{28} »$.

En suivant le deuxième critère, en revanche, la Cour doit utiliser des paramètres externes au sujet, afin d'en vérifier d'abord les convictions puis les actions.

Ces deux critères, face à la notion d'appartenance religieuse, ont trouvé une application ambivalente dans certains arrêts dans lesquels la Cour a évalué la sincérité des valeurs personnelles en fonction $\operatorname{des}^{29}$ ou en alterna-

Mammadov et autres c. Azerbaïdjan; V. W. JEAN-BAPTISTE, «La reconnaissance du droit à l'objection de conscience par la Cour européenne des droits de l'homme», RTDH 2012, p. 671-686; P. MuZNY, «Le droit à l'objection de conscience au service militaire pour motifs religieux: de l'ombre à la lumière », Revue du droit des religions, nº 1, 2016, p. 129. Pour une reconstruction générale de la jurisprudence de la Commission et de la Cour concernant l'objection de conscience, V. D. FERRARI, La libertà di coscienza nella pluralità degli ordinamenti giuridici, Tricase, Libellula Ed., 2015, p. 182.

27. V. G. Di Cosimo, Coscienza e Costituzione, I limiti del diritto di fronte ai convincimenti interiori della persona, Milano, Giuffrè/Francis Lefebvre, 2000, p. 207 et s.

28. V. CEDH, 18 déc. 1996, Valsamis c. Grèce, opinion dissidente commune aux juges Thòr Vilhjálmsson et Jambrekì et CEDH, 18 déc. 1996, Efstratiou c. Grèce, opinion dissidente commune aux juges Thòr Vilhjálmsson et Jambrekì. Dans la première affaire, la Cour a jugé qu'il ne peut être dérogé pour des raisons de conscience ou de religion à l'obligation pour les élèves de participer à la parade annuelle, en mémoire des affrontements entre Grecs et Italiens pendant la Seconde Guerre mondiale, parce que l'événement est de nature pacifique et patriotique. Sur ce point V. Comm. EDH, 3 déc. 1986, Angelini c. Suède.

29. CEDH, 13 avr. 2006, Kosteski c. The Former Yugoslav Republic of Macedonia. 
tive $^{30}$ aux pratiques codifiées par des groupes qui partagent le même credo. Dans l'arrêt Kosteski c. Ex-République yougoslave de Macédoine, la gravité des condamnations a été identifiée par la Cour non pas dans l'analyse des données de la foi individuelle, mais en étudiant la dimension collective de la religion afin de vérifier la légitimité du comportement allégué par le requérant. En particulier, le critère d'appartenance du demandeur à la communauté islamique était considéré principalement dans l'évaluation de la sincérité de l'individu, qui avait justifié son absence au travail pour des raisons liées à la célébration d'une fête religieuse. Le fait que le mode de vie de l'homme ne corresponde pas aux principes de l'islam exprimés par des groupes de la région et qu'il n'avait jamais déclaré publiquement son appartenance religieuse, en changeant de convictions à trois reprises entre 1994 et 2002, a conduit la Cour à ne pas le considérer comme crédible. Les juges de Strasbourg ont finalement identifié dans l'appartenance confessionnelle l'élément qui devait être prouvé par le demandeur pour justifier le traitement spécial requis par l'employeur. Dans ce cas, la gravité de la condamnation n'a donc pas été attribuée en relation avec l'autonomie de l'individu quant à sa façon de vivre sa spiritualité, mais avec les pratiques consacrées par le groupe religieux de référence.

Dans l'arrêt Jakóbski c. Pologne $e^{31}$, la Cour accueille les griefs d'un détenu ${ }^{32}$ qui se plaignait d'une atteinte à sa liberté de religion: la prison, où il purgeait une peine, avait refusé le régime végétarien qu'il souhaitait suivre en tant que bouddhiste. Cette affaire est significative car la Cour a utilisé les notions de conviction et d'appartenance religieuse pour évaluer le rapport entre le requérant et sa religion. En fait, la Cour a jugé que la non-reconnaissance d'un régime végétarien a constitué une violation de l'article 9 de la CEDH, non seulement en raison de la circonstance que «nor was the Buddhist Mission consulted on the issue of the appropriate diet ${ }^{33}$ ", mais également en appliquant les mêmes formules de l'affaire Campbell et Cosans c. Royaume-Uni, pour définir le concept de croyance. En effet, non seulement la « decision to adhere to a vegetarian diet can be regarded as motivated or inspired by a religion ${ }^{34} »$, mais ce choix est l'un des «views that attain a

30. CEDH, 7 déc. 2010, Jakóbski c. Pologne.

31. Ibid.

32. Pour l'appréciation d'une pratique religieuse par le groupe concerné, V. aussi CEDH, 5 déc. 2017, Hamidović c. Bosnie-Herzégovine. V. aussi d'une certaine façon, CEDH, Gde ch., 9 juill. 2013, Sindicatul «Păstorul cel Bun»c. Roumanie.

33. CEDH, Jakóbski c. Pologne, § 52.

34. Ibid., \& 45 . 
certain level of cogency, seriousness, cohesion and importance ${ }^{35}$ ». La même définition de croyance avait cependant déjà été mentionnée par la Cour dans la décision Leela Förderkreis e.V. et autres c. Allemagne ${ }^{36}$ en ce qui concerne les nouveaux mouvements religieux, qui se plaignaient d'une violation de l'article 9, parce que l'État avait manqué à son devoir de neutralité, jugeant le contenu des doctrines professées.

En termes différents, la Grande Chambre de la Cour de Strasbourg, dans la récente décision Molla Sali c. Grèce de décembre $2018^{37}$, a mis en lumière la tension entre la liberté collective de religion et la liberté individuelle de religion dans le contexte de l'appartenance à une minorité religieuse. En fait, les droits garantis aux minorités, s'ils sont appliqués en contradiction avec le choix de l'individu de ne pas appartenir au groupe minoritaire, peuvent devenir une cause de «discrimination par association ${ }^{38} »$. En particulier, dans l'affaire examinée par la Grande Chambre, cette discrimination a eu lieu, puisque la Cour de cassation grecque a établi l'appartenance d'une femme à la minorité musulmane thrace, eu égard au fait que le mari décédé faisait partie de cette communauté. La Cour de Strasbourg a établi qu'un État ne peut imposer à un individu d'appartenir à une minorité religieuse, cette obligation violant «un droit d'importance capitale dans le domaine de la protection des minorités, à savoir le droit de libre identification ${ }^{39}$ ».

35. Ibid., \& 44.

36. CEDH, 6 nov. 2008, Leela Förderkreis e.V. et autres c. Allemagne. Sur le rapport entre la garantie de la liberté religieuse, l'autonomie confessionnelle des groupes religieux et le rôle des États, V. G GonzALEZ, «L'autonomie ecclésiale au risque relatif des droits de l'homme», RTDH 2014, p. 803-818.

37. CEDH, Gde ch., 19 déc. 2018, Molla Sali c. Grèce. V. M. Afroukh, «L'application de la Charia en Grèce: la fermeté incomprise de la Cour européenne des droits de l'homme», RTDH 2019, p. 925-940.

38. Ce concept novateur de discrimination par association a d'abord été examiné par la Cour de justice de l'Union européenne, puis par le Comité des droits des personnes handicapées des Nations unies. La Cour de justice a développé le concept examiné en rapport avec l'origine ethnique (V. CJUE, Gde ch., 16 juill. 2015, C-83/14, CHEZ Razpredelenie Bulgaria $A D$ c. Komisia za zashtita ot diskriminatsia), tandis que le Comité des Nations unies a défini ce concept en rapport avec le handicap (V. Comité des droits des personnes handicapées, Observation générale $n^{\circ} 6$ (CRPD/C/GC/6), 26 avr. 2018, § 20), en soutenant que: «La discrimination "fondée sur le handicap" peut viser [...] les personnes qui sont associées à une personne handicapée. Cette dernière forme de discrimination est dite "discrimination par association" ». La Cour de Strasbourg a appliqué ce concept aux minorités religieuses dans l'affaire Molla Sali c. Grèce. En effet, la Cour a précisé dans sa jurisprudence que: «C'est la première fois que la Grande Chambre examine la question et conclut à l'existence d'une discrimination par association. Autrement dit, la violation de l'article 14 en combinaison avec l'article 1 du Protocole $\mathrm{n}^{\circ} 1$ est établie à raison non pas de la religion de la requérante mais de celle de son époux, de confession musulmane »: CEDH, Gde ch., 19 déc. 2018, Molla Sali c. Grèce, \& 7.

39. Ibid., \& 157. 


\section{LE JUGEMENT SUR LES CROYANCES ET CONVICTIONS PERSONNELLES: L'IMPACT DU CONTEXTE NATIONAL}

Avant d'analyser la jurisprudence de la Cour de Strasbourg, il semble nécessaire de définir le concept de contexte national utilisé dans cette réflexion et la relation entre ce concept et celui d'appartenance religieuse.

Le contexte national décrit l'impact de la provenance nationale du recours sur la protection de la liberté de religion, eu égard à l'application de l'article $9 \& 2$ de la CEDH. La Cour de Strasbourg, sous certaines conditions, ne juge pas la conformité des différentes législations nationales à l'égard de la Convention. Cette circonstance semble établir, bien qu'implicitement, un lien entre le requérant et le contexte juridique et national à partir duquel provient l'appel.

Passé le test de sérieux et de bonne foi, les expressions des convictions personnelles ne doivent pas être contraires aux limites fixées à l'alinéa 2 de l'article 9, limites qui, en vertu du même article 9, doivent contribuer aux besoins propres à une société démocratique.

D'une analyse globale de la jurisprudence de Strasbourg, on peut distinguer un modèle européen de société démocratique ${ }^{40}$ construit sur certains principes fondamentaux: le respect de la dignité humaine, qui est «l'essence même» de la Convention ${ }^{41}$; la primauté du droit, à laquelle le préambule fait expressément référence et qui représente «un des principes fondamentaux d'une société démocratique [...] dont s'inspire la Convention tout entière ${ }^{42} »$; le pluralisme, qui est «consubstantiel» à la vie démocratique ${ }^{43}$; le principe de non-discrimination, qui «constitue l'un des principes fondamentaux de la démocratie ${ }^{44} »$; le respect des droits des autres ${ }^{45}$.

40. Dans ce sens, V. amplius, Fl. JACQUemot, Le standard européen de société démocratique, Montpellier, Fac. droit de Montpellier, 2006.

41. CEDH, 22 nov. 1995, S.W. c. Royaume-Uni. En particulier, la protection de la liberté de conscience est profondément liée à la protection de la dignité humaine. En effet, si tous les hommes ont une conscience, la protection de la liberté morale veille à ce que la valeur de la dignité corresponde non seulement à tous les hommes, mais surtout à chaque individu.

42. CEDH, 29 nov. 1988, Brogan et autres c. Royaume-Uni, § 58.

43. CEDH, Kokkinakis c. Grèce, précit., § 31. V. G. GonZALEZ, «Pluralisme et religions dans la jurisprudence de la Cour européenne des droits de l'Homme», in Droit et religion en Europe, Strasbourg, Presses universitaires de Strasbourg, 2014, p. 131-141.

44. CEDH, Gde ch., 13 févr. 2003, Refah Partisi et autres c. Turquie, § 119.

45. CEDH, Gde ch., $1^{\mathrm{er}}$ juill. 2014, S.A.S. c. France. 
Par rapport à ces principes, la Cour a reconnu au cours du temps les différentes traditions nationales ${ }^{46}$, réservant une marge d'appréciation aux États ${ }^{47}$. En effet, le juge européen, notant l'absence d'un dénominateur commun entre les États membres sur la liberté de conscience et de religion ${ }^{48}$, leur a reconnu une large marge d'appréciation, examinant en de rares occasions les principes constitutionnels qui dans chaque État définissent le cadre général des relations entre les pouvoirs publics et les convictions personnelles ${ }^{49}$. Dans ces cas, le besoin démocratique de limiter la liberté morale est influencé par l'appartenance des demandeurs à une communauté spécifique de l'État.

Le jugement du besoin qui détermine la légitimité des limites aux libertés garanties par l'article 9 peut donc changer en fonction du degré d'incidence que la Cour de Strasbourg reconnaît au droit national. Par conséquent, en fonction du contexte national et de l'objet du recours, les contenus des clauses restrictives de la loi nationale en question peuvent changer. En effet, si la Cour ramène «la notion de nécessité [à] un besoin social impérieux [...] la mesure prise doit être proportionnée au but légitime poursuivi ${ }^{50} »$, la proportionnalité de la restriction sera plus ou moins discutée en fonction

46. Cette reconnaissance identifie également le concept de pluralisme international, V. M. Delmas Marty, "Pluralisme et traditions (revendication des droits individuels)», in P. TAVERnier (dir.), Quelle Europe pour les droits de l'homme? La Cour de Strasbourg et la réalisation d'une "Union plus étroite», Bruxelles, Bruylant, 1996, p. 81 et s.

47. V. F. Sudre, «Le pluralisme saisi par le juge européen», in M. Levinet (dir.), Pluralisme et juges européens des droits de l'homme, Bruxelles, Bruylant, 2010, p. 51-52. La Cour de Strasbourg ne souhaite pas remplacer les autorités nationales compétentes, en raison du caractère subsidiaire du système de protection des droits établi par la CEDH; V. CEDH, 23 juill. 1968, Affaire linguistique belge, \& 10. Le juge européen protège donc le pluralisme international, en l'identifiant dans la «reconnaissance et le respect réel de la diversité et des dynamiques des traditions culturelles, des identités ethniques et culturelles, des convictions religieuses et des idées et concepts artistiques, littéraires », ainsi CEDH, Gde ch., 17 janv. 2004, Gorzelik et autres c. Pologne, § 92; de même, CEDH, 26 avr. 1979, Sunday Times c. Royaume-Uni, § 61. Plus généralement, donc, la Convention ne doit pas être considérée comme un instrument de garantie des droits et des libertés destiné à ignorer la spécificité des systèmes nationaux ou à se substituer à la législation nationale; V. G. Cohen-Jonathan, «La Convention européenne des droits de l'homme socle de la protection des droits de l'homme dans le droit constitutionnel européen », in R.-J. Dupuy (dir.), Mélanges Valticos, Paris, Pédone, 1999, p. 227-263.

48. Par ex., le juge européen a constaté qu'il n'existe pas en Europe de «notion uniforme de morale» (CEDH, 7 déc. 1976, Handyside c. Royaume-Uni, § 48) ou « une conception uniforme de la signification de la religion dans la société»(CEDH, 20 sept. 1994, OttoPreminger Institut c. Autriche, \& 50).

49. La Cour, par exemple, ne juge pas les interdictions découlant du choix laiqque d'un système incompatibles avec la protection de la liberté de conscience (V., ex plurimis, CEDH, 4 déc. 2008, Dogru c. France et Kervanci c. France) de même pour la présence de symboles religieux à l'école (V. CEDH, Gde ch., 18 mars 2011, Lautsi c. Italie).

50. Comm. EDH, 24 nov. 1986, Gillow c. Royaume-Uni. 
du poids accordé à la marge nationale d'appréciation. La marge nationale d'appréciation exprime une dynamique dans laquelle la Cour reconnaît aux États la possibilité d'encadrer les libertés consacrées par la Convention ${ }^{51}$. De cette façon, les résultats des différents processus historiques de définition des rapports entre le sacré et le profane, dans chaque État, réapparaissent dans la protection conventionnelle des libertés en question ${ }^{52}$.

En particulier, les limites de l'ordre public et de la moralité publique ne permettent pas d'identifier des concepts similaires dans les États membres de la Convention, et cela pour deux raisons: l'une liée aux différentes traditions juridiques auxquelles appartiennent les pays européens ${ }^{53}$; l'autre liée à l'impossibilité, jusqu'à présent observée, de donner une définition systématique du modèle de neutralité des pouvoirs publics dans leur rapport avec les différentes convictions ou croyances ${ }^{54}$.

La solution proposée dans la Convention semble être la détermination de la notion administrative d'ordre public ${ }^{55}$ comme situation concrète de paix sociale, où chacun peut jouir de ses droits sans déranger l'autre. Proche de

51. En ces termes, le concept de marge d'appréciation est apparu dans les réflexions sur le principe de subsidiarité, en tant que critère régissant l'application de la Convention par la Cour et les États, et a été inclus dans la Déclaration de Brighton en 2012. Dans cette déclaration, qui exprime les résultats de la Conférence tenue à Brighton les 19 et 20 avril 2012 à l'initiative de la présidence britannique du Comité des ministres du Conseil de l'Europe, on affirme en effet que «La jurisprudence de la Cour indique clairement que les États parties disposent, quant à la façon dont ils appliquent et mettent en œuvre la Convention, d'une marge d'appréciation qui dépend des circonstances de l'affaire et des droits et libertés en cause. Cela reflète le fait que le système de la Convention est subsidiaire par rapport à la sauvegarde des droits de l'homme au niveau national et que les autorités nationales sont en principe mieux placées qu'une Cour internationale pour évaluer les besoins et les conditions au niveau local. La marge d'appréciation va de pair avec la supervision découlant du système de la Convention. À cet égard, le rôle de la Cour est d'examiner si les décisions prises par les autorités nationales sont compatibles avec la Convention, eu égard à la marge d'appréciation dont dispose [sic] les États»; V. Conférence sur l'avenir de la Cour européenne des droits de l'homme, Déclaration de Brighton, B. Interaction entre la Cour et les autorités nationales, p. 3, https://www.echr. coe.int/Documents/2012_Brighton_FinalDeclaration_FRA.pdf [consulté le 20 janv. 2020].

52. CEDH, 20 sept. 1994, Otto Preminger Institut c. Autriche.

53. Du premier point de vue, dans le texte français de la Convention se trouve l'expression d'ordre public qui dans le droit français, appartenant à la famille juridique de la civil law, identifie des concepts tels que: paix publique, ordre, maintien de l'ordre, protection de l'ordre. En revanche, dans la version anglaise la référence à l'ordre public, comme limite à la libre expression des croyances ou convictions religieuses, renvoie au public order, qui, dans les systèmes de common law, identifie le manque de désordre et donc l'équilibre d'une société ordonnée. V. F. SUDRE (dir.), Les grands arrêts de la Cour européenne des droits de l'homme, Paris, PUF, 8e éd. 2019.

54. Ibid.

55. Ainsi F. Margiotta Broglio, La protezione internazionale della libertà religiosa nella convenzione europea dei diritti dell'uomo, Milano, Giuffrè, p. 60 et s. 
cette notion matérielle, l'idéal est représenté par le fait que la paix sociale répond aux besoins d'une société démocratique ${ }^{56}$ et donc que l'État ne porte aucun jugement de valeur sur les convictions personnelles pour en déterminer l'opposition à l'ordre public ou à la morale publique, mais assure que «les groupes concurrents se tolèrent les uns les autres ${ }^{57}$ ».

Toutefois, la neutralité des différents États n'étant pas univoque, l'ordre public et la morale publique reflètent les normes fondamentales éthiques et politiques sur lesquelles se fonde chaque loi nationale, expression des différents systèmes de rapports entre la sphère temporelle et la sphère spirituelle. En effet, le contenu juridique varié des modèles de rapport entre États et confessions religieuses ${ }^{58}$ se reflète également sur les contenus de valeur pris en considération et protégés sur le plan positif, à travers des principes comme l'ordre public ${ }^{59}$ ou la morale publique. On retrouve donc, au sein des différents États européens, un contenu différent de garantie de la liberté de conscience et de religion.

Par exemple, le modèle laïque d'ordre public se développe dans les États qui ont constitutionnalisé la neutralité de l'État à travers le principe de laïcité, comme en France, en Turquie ${ }^{60}$ et en Suisse ${ }^{61}$, où les pratiques devraient

56. V. C. PIŠTAN, «La libertà religiosa nella giurisprudenza della Corte europea dei diritti dell'uomo», in L. Mezzetti, A. Morrone (dir.), Lo strumento costituzionale dell'ordine pubblico europeo. Nei sessant'anni della Convenzione per la salvaguardia dei diritti dell'uomo e delle libertà fondamentali (1950-2010), Torino, Giappichelli, 2012, p. 353 et s.

57. CEDH, 14 déc. 1999, Serif c. Grèce, § 55.

58. V. B. Basdevant-Gaudemet, F. Messner, Les origines historiques du statut des confessions religieuses dans les pays de l'Union européenne, Paris, PUF, 1999, passim et F. Messner, «Les rapports entre les Églises et les Etats en Europe: la laïcité comme modèle?», in G. Gonzalez (dir.), Laïcité, liberté de religion et Convention européenne des droits de l'homme, Bruxelles, Bruylant, 2006, p. 51 et s.

59. Traditionnellement, l'ordre public peut être décliné suivant deux grands modèles: 1) ordre public matériel qui restreint certains phénomènes en raison de leurs effets concrets, afin de protéger la sécurité publique; 2) ordre public immatériel, exprimant une approche juridique visant à défendre certaines valeurs, choisies par la majorité de la société. V. M.-J. REDOR (dir.), L'ordre public: ordre public ou ordres publics? Ordre public et droits fondamentaux. Actes du colloque de Caen, 11 et 12 mai 2000, Bruxelles, Bruylant, 2001.

60. Dans ce système, le principe de laïcité est appliqué comme un mécanisme de laïcité active qui ne régit pas uniquement les rapports entre les confessions religieuses et l'État, mais réglemente également «la vie sociale, l'éducation, la famille, l'économie, le droit, les mœurs, les codes de conduite», ce qui représente une véritable philosophie de vie. V. Cour const. turque, 16 janv. 1997, nº 1998/1. D'intérêt pour le sujet, A. T. KuRU, Secularism and State Polices toward religion. The United States, France and Turkey, Cambridge, Cambridge University Press, 2009, p. 161 et s.

61. Par ex., CEDH, déc. 15 févr. 2011, Dahlab c. Suisse : V. M. Ciravegna, « La nozione di "segno esteriore forte" tra problemi di definizione e presunzione di lesività: la sentenza "Dahlab c. Svizzera" ", in R. Mazzola (dir.), Diritto e religione in Europa, Bologna, Il Mulino, 2012, p. 141 et s. 
être mises en balance avec ce principe, n'étant pas nécessairement considérées comme protégées par l'article $9^{62}$ lorsqu'elles sont limitées par des règles juridiques mettant en œuvre le principe de laïcité. Dans un arrêt récent, la Cour a jugé que l'interdiction imposée en France de circuler à visage couvert, tout en représentant une ingérence dans la liberté de religion d'une femme qui avait l'habitude de porter la burqa dans l'espace public, est une expression légitime de la marge d'appréciation accordée aux autorités de l'État ${ }^{63}$. La Cour a en effet précisé que dans une société démocratique, «le voile cachant le visage [est perçu] par l'État défendeur comme portant atteinte au droit d'autrui d'évoluer dans un espace de sociabilité facilitant la vie ensemble ${ }^{64} »$. Avec ce raisonnement, la Cour semble avoir accepté la définition d'ordre public immatériel ${ }^{65}$ élaborée par le gouvernement français dans le «projet de loi interdisant la dissimulation du visage dans l'espace public» de $2010^{66}$. Dans l'étude d'impact de la loi, en effet, l'interdiction de circuler avec la burqa était justifiée, au-delà de la défense de l'ordre public matériel, par la nécessité de s'opposer à un comportement «contraire aux exigences fondamentales du "vivre ensemble" dans la société française». En ces termes, dans une société démocratique, même les formes de manifestation de la liberté religieuse qui ne mettent pas en péril la préservation de la sécurité, de l'ordre, de la santé ou de la morale publiques, peuvent être limitées si elles s'opposent «aux droits [...] d'autrui ${ }^{67} »$ de vivre ensemble. Cette interprétation de la Cour semble être novatrice puisque les règles sociales prévalant dans un État sont considérées comme l'objet d'un droit dont la garantie peut déterminer la nécessité démocratique de limiter, en vertu de l'article $9 \& 2 \mathrm{CEDH}$, les pratiques religieuses considérées en conflit. Même

62. V. CEDH, 4 déc. 2008, Dogru c. France et Kervanci c. France, \& 69.

63. V. le dossier sur «La dissimulation du visage dans l'espace public», Revue du droit des religions, $\mathrm{n}^{\circ}$ 2, 2016; G. GONZALEZ, G. HAARSCHER, «Consécration jésuitique d'une exigence fondamentale de la civilité démocratique? Le voile intégral sous le regard des juges de la Cour européenne», RTDH 2015, p. 219-233.

64. CEDH, Gde ch., S.A.S. c. France, précit., § 122. En ce sens, V. CEDH, 12 juill. 2017, Dakir c. Belgique. Sur la notion de "vivre ensemble» V. G. GonzAlEz, «La notion de "vivre ensemble" dans la jurisprudence de la Cour européenne des droits de l'homme relative à la liberté de religion», Quad. dir. pol. eccl, 1, 2016, p. 99-110.

65. Sur la notion d'ordre public immatériel, V. B. STIRN, «Ordre public et libertés publiques», intervention lors du colloque sur l'ordre public organisé par l'Association française de philosophie du droit, 17 sept. 2015: https://www.conseil-etat.fr/actualites/discours-etinterventions/ordre-public-et-libertes-publiques [consulté le 20 janv. 2020].

66. Projet de loi interdisant la dissimulation du visage dans l'espace public. Étude d'impact, mai 2010: http://www.assemblee-nationale.fr/13/projets/pl2520-ei.asp [consulté le 20 janv. 2020].

67. V. Art. $9 \S 2$ CEDH. 
en ce qui concerne le concept de moralité publique, la Cour a fait remarquer qu'«il n'y a pas encore, dans les ordres juridiques et sociaux des États membres du Conseil de l'Europe, une concordance de vues suffisante pour conclure qu'un système permettant à un État d'imposer des restrictions à la propagation d'articles réputés blasphématoires n'est pas en soi nécessaire dans une société démocratique ${ }^{68} »$. Le concept de sécurité publique était qualifié par la Cour comme garantie de paix sociale, en relation avec les phénomènes de subversion et de terrorisme ${ }^{69}$ qui, menaçant l'existence d'une société démocratique, portent atteinte à l'exercice des droits individuels ${ }^{70}$.

La prévalence du critère de contexte national sur l'autonomie de la personne émerge également dans le sujet délicat de la liberté de choix éducatif, religieux ou philosophique, en famille ${ }^{71}$, par exemple concernant la présence de symboles religieux à l'école. Donc, compte tenu de la reconnaissance des différentes traditions nationales, une étudiante ne pourra pas porter le voile dans une université publique turque ${ }^{72}$, elle pourra le faire dans un lycée public italien où un crucifix sera également accroché au mur ${ }^{73}$, contrairement à une école publique française où la croix ne pourra être ni accrochée ni portée par les enseignants ou les élèves si elle est considérée comme une manifestation ostentatoire des convictions religieuses ${ }^{74}$.

68. Ainsi, CEDH, 25 nov. 1996, Wingrove c. Royaume-Uni, \& 57.

69. Sur les rapports entre sécurité et liberté, en relation avec les phénomènes terroristes, V. G. De Vergottini, «La difficile convivenza fra libertà e sicurezza. La risposta delle democrazie al terrorismo », Rassegna parlamentare 2004, p. 439 et s; S. FERRARI, «Libertà religiosa e sicurezza nazionale in Europa dopo l'11 settembre», Quad. dir. pol. eccl., 1, 2005, p. 123 et s.; A. Lambert, L. Braconnier Moreno, «La marge de manœuvre de la France dans le déclenchement d'un régime dérogatoire aux libertés fondamentales, une dénaturation de l'article 15 de la CEDH? », La Revue des droits de l'homme, 2016: https:// journals-openedition-org.scd-rproxy.u-strasbg.fr/revdh/1778 [consulté le 20 janv. 2020]. Dans une autre perspective, en ce qui concerne la relation entre le droit d'asile et le terrorisme, V. D. FERRARI, "Religion and Terrorism in International Protection», in Sekcia Medzinárodného a európskeho práva. Vplyv globalizàcie na medzinárodné a európske legislativne procesy, Banskey Bistrici, Belianum, 2019, p. 61-73.

70. Ce besoin est confirmé par la Cour de Strasbourg dans CEDH, Gde ch., 27 sept. 1995, McCann et autres $c$. Royaume-Uni: A. CASSESE, I diritti umani oggi, Roma-Bari, Laterza, 2005, p. 202 et s. V. aussi CEDH, 29 avr. 2019, A.M c. France.

71. Ce droit est garanti par l'art. 2 du Protocole $n^{\circ} 1$ à la Convention.

72. CEDH, Gde ch., 10 nov. 2005, Leyla Sahin c. Turquie; V. J. F. Flauss, « Le port de signes religieux distinctifs par les usagers dans les établissements publics d'enseignement », in G. Gonzalez (dir.), Laïcité, liberté de religion..., op. cit., p. 201 et s.

73. CEDH, Gde ch., 18 mars 2011, Lautsi c. Italie, § 36.

74. CEDH, déc. 30 juin 2009, Aktas c. France, à propos de la loi no 2004-228 du 15 mars 2004 encadrant, en application du principe de laicité, le port de signes ou de tenues manifestant une appartenance religieuse dans les écoles, collèges et lycées publics. 


\section{CONCLUSIONS}

Si la qualification juridique du facteur religieux a historiquement été caractérisée par le principe d'appartenance à une même communauté et donc au respect des règles, pratiques et rites décidés par le groupe, avec la proclamation internationale de la liberté de conscience et de religion, la reconnaissance du libre arbitre dans le domaine moral a inversé cette tendance. La personne doit être protégée dans ce qui relève de sa liberté, indépendamment du partage de valeurs spécifiques avec les autres. En effet, la liberté de conscience et de religion garantit également le choix de ne pas adhérer à une communauté ou de la contester tout en en faisant partie ${ }^{75}$.

Pour évaluer si un comportement exprime les croyances ou les convictions de la personne, la Cour de Strasbourg a créé un double examen qui, dans certains cas, a imposé aux requérants une limitation de leur libre arbitre fondé sur un double jugement d'appartenance. En effet, la nature morale du comportement a d'abord été estimée sur des critères hétéronomes, à savoir l'existence d'un lien entre l'individu et un groupe religieux ou philosophique. Lorsque cette évaluation est avérée (le comportement correspond à des pratiques codifiées par des formations sociales), la légitimité des limites imposées est déterminée sur la base de la marge nationale d'appréciation et, par conséquent, d'appartenance de la personne à un modèle national spécifique de rapport entre les autorités et les confessions ou croyances personnelles. Cette appartenance, lorsqu'elle tend à un principe d'autorité, comme cela a été par le passé, risque de limiter les libertés humaines dans le domaine moral. 